\title{
Integral data assimilation of the MERCI-1 experiment for the nuclear data associated with the PWR decay heat computation
}

\author{
J. Huyghe ${ }^{1, *}$, C. De Saint-Jean ${ }^{1}$, D. Lecarpentier ${ }^{2}$, C. Reynard-Carette ${ }^{3}$, C. Vaglio- \\ Gaudard $^{1}$, V. Vallet ${ }^{1}$. \\ ${ }^{1}$ CEA, DEN, DER Cadarache, F-13108 Saint Paul les Durance, France \\ ${ }^{2}$ EDF Research and Development, 7 Boulevard Gaspard Monge, Palaiseau, France \\ ${ }^{3}$ Aix Marseille Univ, Université de Toulon, CNRS, IM2NP, Marseille, France
}

\begin{abstract}
Nuclear decay heat is a crucial issue for PWR in-core safety after reactor shutdown and back-end cycle. It is a dimensioning parameter for safety injection systems (SIS) to avoid a dewatering of the reactor core. The decay heat uncertainty needs to be controlled over the largest range of applications. The assimilation of the MERCI-1 experiment was studied to provide feedbacks on nuclear data. This experiment consisted in the measurement of the decay heat of a PWR UOX fuel sample irradiated in the OSIRIS reactor, for cooling times between 45 minutes and 42 days. More specifically, the consideration of several experimental values of MERCI-1 at different cooling times was tested. This raised issues about correlations to consider between different measurements. Besides, the impact of considering correlations between independent fission yields in covariance matrices on the decay heat uncertainty calculation and on the feedbacks on nuclear data is discussed.
\end{abstract}

\section{Introduction}

Decay heat is released by both radioactive decay of unstable fuel and material structure isotopes after reactor shutdown. It is a key challenge for in-core safety and back-end cycle. The decay heat depends on about 40,000 nuclear data, and mainly from fission yields. With the currently used JEFF-3.1.1 nuclear data evaluation, independent fission yield uncertainties are strong (mainly between 10 and $35 \%$ at 1 standard deviation (std)) and no correlation matrices are associated with the independent fission yields. New evaluations are proposed in the JEFF-3.3 evaluation, recently released. In the framework of nuclear data adjustment, the assimilation of the MERCI-1 integral experiment [1] for a feedback on the nuclear data involved in the nuclear decay heat calculation is discussed in this paper. In particular, the impact of considering correlations between independent fission yields [2] on the integral data assimilation process is studied. The second aspect discussed in the paper is the use of several measurements from the MERCI- 1 experiment at the same time in the assimilation process. To do this, an experimental correlation matrix needs to be determined.

*Corresponding author: jordan.huyghe@cea.fr 


\section{MERCI-1 experiment overview}

The MERCI-1 integral experiment [1] represents a significant contribution for the experimental validation of short cooling time decay heat calculation tools. Carried out in 2008 , it consisted in irradiating a PWR UOX fuel rod sample $\left(3.7 \mathrm{w} . \%{ }^{235} \mathrm{U}\right.$ enrichment) in the French OSIRIS experimental reactor (CEA Saclay, France), and then in measuring the decay heat released by the sample with the MOSAIC calorimeter [3]. The OSIRIS reactor core was loaded with $\mathrm{U}_{3} \mathrm{Si}_{2} \mathrm{Al}$ plates $\left(\mathrm{e}_{235 \mathrm{U}}=19.75 \%\right)$. The burnup of the sample reached at the end of irradiation was $3.6 \mathrm{GWd} / \mathrm{t}$. The MOSAIC calorimeter is based upon the heat pipe principle. The decay heat is assessed by a heat balance measurement on the secondary system using an instrumentation designed to have a decay heat uncertainty of $0.5 \%$ at $1 \mathrm{std}$ and its measurement goes from 45 minutes to 42 days of cooling time.

\section{Control of the uncertainties associated with the $\mathrm{MERCl}-1$ experiment}

To be used within the framework of the integral assimilation (cf. section 4), both the experimental uncertainty associated with MERCI-1 and the calculation uncertainty associated with the interpretation of the experiment need to be rigorously estimated and controlled.

\subsection{MERCI-1 experimental uncertainty}

Different sources of experimental uncertainties can be identified, related to the use of the MOSAIC calorimeter [3] for the measurement of the irradiated fuel's decay heat. The sources are the temperature difference between the inlet and outlet of the condenser of the calorimeter $(\Delta T)$, the heat capacity of water $\left(C_{p}\right)$, the water mass flow measured between the inlet and outlet of the condenser $\left(\mathrm{Q}_{\mathrm{m}}\right)$. All these sources of uncertainties are propagated to the decay heat uncertainty. The decay heat is obtained through the formula (1) as the image of the enthalpy balance between the inlet and the outlet of the condenser:

$$
D H=Q_{m} \mathrm{C}_{\mathrm{p}} \Delta \mathrm{T}
$$

The different sources of experimental uncertainty are assumed independent: thus the global uncertainty is obtained by combining them in a quadratic summation. The different terms in the quadratic summation are the products of the sensitivity of the decay heat to each term in equation (1) with the relative uncertainty of each parameter $\left(\mathrm{Q}_{\mathrm{m}}, \mathrm{C}_{\mathrm{p}}, \Delta \mathrm{T}\right)$, given the fact that there are no correlations between $\mathrm{Q}_{\mathrm{m}}$ and $\mathrm{C}_{\mathrm{p}}$ and between $\mathrm{Q}_{\mathrm{m}}$ and $\Delta \mathrm{T}$, and that the correlation between $\mathrm{C}_{\mathrm{p}}$ and $\Delta \mathrm{T}$ was determined and is negligible. The resulting uncertainty on the decay heat is $0.5 \%$ at $1 \mathrm{std}$, for cooling times between 45 minutes and 42 days except between 16 and 21 days and between 23 and 25 days where it is $1.0 \%$ at 1 std, due to a valve failure leading to a discontinuity in the heat measurement.

\subsection{MERCI-1 calculation uncertainty}

The calculation uncertainty associated with the MERCI-1 experiment comes from different sources:

- the gamma leakage and activation of the cladding and overcladding: the gamma leakage was determined with a stochastic code and the activation of the cladding was 
taken into account in the decay heat calculation. The main contributors to the gamma decay heat and activation power for the cooling times considered are ${ }^{140,142} \mathrm{La},{ }^{132,134} \mathrm{I}$ and ${ }^{138} \mathrm{Cs}$ for the former (responsible for the gamma leakage given their gamma spectra) and ${ }^{56} \mathrm{Mn}$, ${ }^{60} \mathrm{Co}$ and ${ }^{51} \mathrm{Cr}$ for the latter. Judging from these isotopes' associated nuclear data uncertainties (gamma decay energies and periods), the resulting uncertainties on the gamma leakage and activation power of the cladding and overcladding is negligible with respect to the other sources of uncertainty.

- the adjustment of the burnup reached at the end of irradiation (the adjustment was done by minimizing the calculation-over-experiment discrepancies of the neodymium concentrations $\left({ }^{145,146,148,150} \mathrm{Nd}\right)$; this adjustment depends on the cumulated fission yield uncertainties of ${ }^{235} \mathrm{U}$ to the neodymium isotopes. The resulting uncertainty is $1.1 \%$ at 1 std on the decay heat.

- the irradiation conditions (fuel temperature, coolant temperature and ${ }^{235} \mathrm{U}$ initial enrichment whose uncertainties are evaluated by direct perturbation in the transport calculation and results in in a global uncertainty of $0.1 \%$ at 45 minutes of cooling and $1.1 \%$ at 42 days of cooling at 1 std).

The sources of calculation uncertainties together with the experimental uncertainties for the MERCI-1 experiment were propagated to the decay heat by quadratic summation, assuming that they are all independent, and result in a global decay heat uncertainty going from 1.2 to $1.6 \%$ at $1 \mathrm{std}$ according to the cooling time considered.

\section{Integral data assimilation of the MERCI-1 experiment}

\subsection{CONRAD code}

The evaluation code used for the data assimilation of the MERCI-1 experiment is CONRAD (COde for Nuclear Reaction Analysis and Data assimilation), developed at CEA, Cadarache [4]. CONRAD enables, inter alia, the assimilation of integral experiments to provide feedback on nuclear data. It is based on a prior set of nuclear data that will be updated through the assimilation of the integral data. The adjustment is done within the framework of the Bayesian inference, by minimizing the Generalized Least Squared function (cf. equation (2)), giving the posterior value of each parameter $\mathrm{x}$ fitted (bias adjustment), and their posterior covariances $\mathrm{M}_{\mathrm{x}}{ }^{\mathrm{p}}[4]$, that will be referred to as trends in the paper.

$$
\mathrm{S}(\mathbf{x})=\left(\mathbf{x}-\mathbf{x}_{\mathbf{0}}\right)^{\mathrm{T}} \mathrm{M}_{\mathbf{x}}{ }^{-1}\left(\mathbf{x}-\mathbf{x}_{\mathbf{0}}\right)+(\mathbf{C}(\mathbf{x})-\mathbf{E})^{\mathrm{T}} \mathrm{M}_{\mathrm{C} / \mathrm{E}}{ }^{-1}(\mathbf{C}(\mathbf{x})-\mathbf{E})
$$

where $\mathbf{x}$ are the parameters (nuclear data), $\mathbf{x}_{\mathbf{0}}$ are the prior values of the parameters, $\mathrm{M}_{\mathrm{x}}{ }^{0}$ is the prior covariance matrix of the parameters, $\mathbf{C}(\mathbf{x})$ are the calculation values of the decay heat (function of the nuclear data, which explains why sensitivities of the decay heat to nuclear data need to be computed), $\mathbf{E}$ are the experimental values of the decay heat considered for the adjustment, and $\mathrm{M}_{\mathrm{C} / \mathrm{E}}$ is the experimental covariance matrix, containing the propagated calculation and experimental $(\mathrm{C} / \mathrm{E})$ decay heat uncertainties.

\subsection{Assimilation of one particular MERCI-1 experimental value}

Let us consider the case of a standard PWR UOX fuel irradiated up to $15 \mathrm{GWd} / \mathrm{t}$ (about one cycle in reactor) at around one year of cooling (for fuel transport issues for instance). Within the framework of the adjustment of nuclear data of importance for the decay heat 
calculation, the first study consisted in assimilating the last measurement of the MERCI-1 experiment, at 42 days of cooling, whose associated calculation/experiment discrepancy $(\mathrm{C} / \mathrm{E}-1)$ is $-0.83 \% \pm 1.6 \%$ (calculation performed with the DARWIN2.3 package [5] and the JEFF-3.1.1 nuclear data evaluation [6]). Sensitivity studies performed on the MERCI-1 fuel rod meshing and the number of depletion steps showed that the biases due to the calculation scheme are negligible [1]. Therefore, it is possible to use the integral data assimilation theory with the MERCI-1 C/E discrepancies. The list of parameters to fit with CONRAD to perform the adjustment was established from the major parameters the decay heat was sensitive to, at 42 days of cooling for the MERCI-1 measurement, and at around 1 year of cooling for the PWR UOX fuel. The corresponding parameters are the ${ }^{235} \mathrm{U}$ thermal fission cross section and the independent thermal fission yields of ${ }^{235} \mathrm{U}$ to ${ }^{140} \mathrm{Xe},{ }^{140} \mathrm{Cs},{ }^{95} \mathrm{Sr}$, ${ }^{95} \mathrm{Y},{ }^{144} \mathrm{La}$, and ${ }^{144} \mathrm{Ba}$. The results of the adjustment for these parameters are shown in Fig. 1. The JEFF-3.1.1 uncertainty values of each parameter are drawn in green on Fig. 1. The trends for each parameter obtained with CONRAD (respectively planned with JEFF-3.3 [7]) are plotted in blue (respectively red). The results show that no actual significant changes are observed with the use of one experiment, except for the uncertainties of the fission yields of ${ }^{235} \mathrm{U}$ to ${ }^{140} \mathrm{Cs}$ and ${ }^{95} \mathrm{Y}$ which are reduced. However, the trends obtained are consistent with JEFF-3.3 at 1 std.

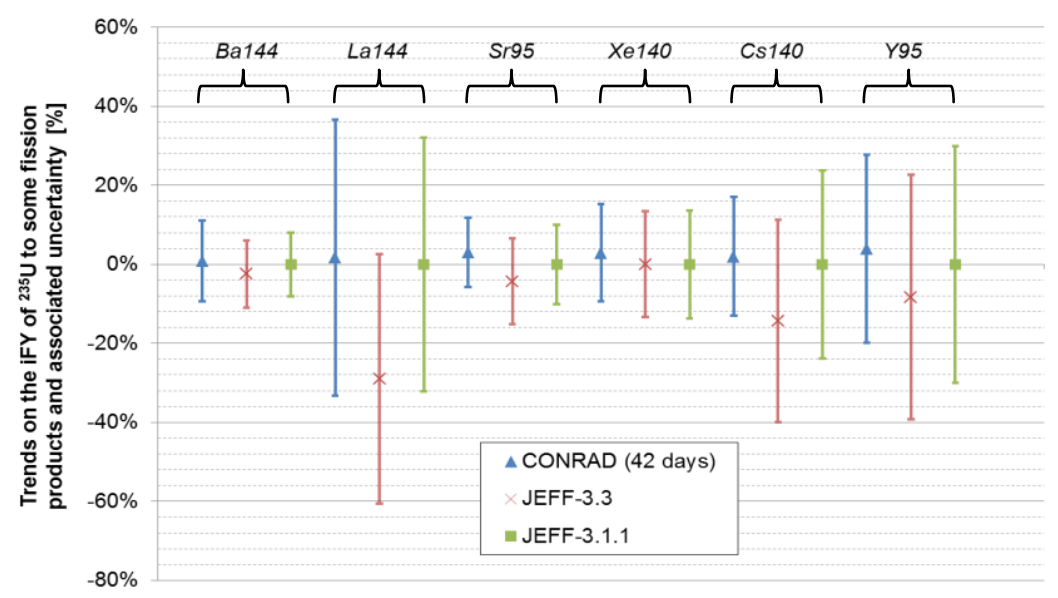

Fig. 1. Feedbacks on JEFF-3.1.1 independent fission yield (iFY) data after assimilation of the MERCI-1 experimental at 42 days of cooling with CONRAD and comparison to JEFF-3.3 trends

\subsection{Impact of the use of correlations between independent fission yield data}

The main nuclear data for the decay heat computation are fission yield data. JEFF-3.1.1 does not provide covariance matrices for independent fission yields, therefore covariance matrices for both fissile systems of ${ }^{235} \mathrm{U}$ and ${ }^{239} \mathrm{Pu}$ were produced at the CEA, associated with JEFF-3.1.1 fission yield data [2]. The covariance matrices produced are stored in the COMAC covariance matrix database [8]. Without these correlations, the decay heat uncertainty at around one year of cooling for the standard PWR UOX fuel is around 4\%. When using these correlations, the decay heat uncertainty is reduced to $1 \%$. The results of using these correlations in the integral data assimilation of the MERCI-1 experimental value mentioned in subsection 4.2 are shown in Fig. 2, compared to JEFF-3.3 and the assimilation previously performed without correlations between independent fission yield data (cf. subsection 4.2). Results show that using correlations between independent fission yields enables more consistent trends with JEFF-3.3 of the fission yield data of ${ }^{235} \mathrm{U}$ to the major contributors of the decay heat at the cooling times considered. In particular, the 
trends obtained for the independent fission yields of ${ }^{235} \mathrm{U}$ to ${ }^{144} \mathrm{La}$ and ${ }^{140} \mathrm{Cs}$ are more in accordance with JEFF-3.3 than what was obtained in Fig. 1. Moreover, the associated uncertainties of these fission yields are reduced.

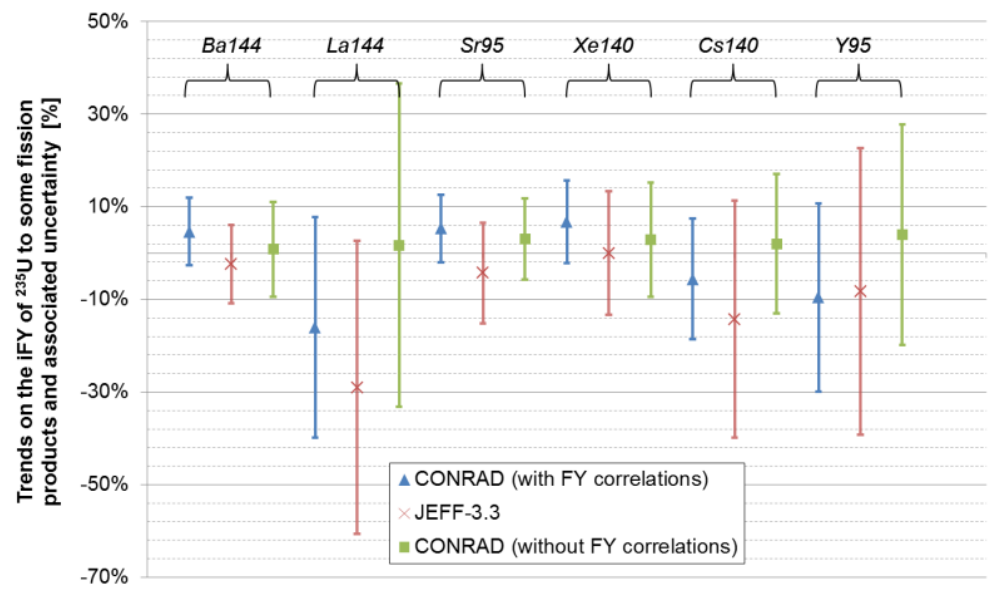

Fig. 2. Feedbacks on JEFF-3.1.1 correlated iFY data after assimilation of one MERCI-1 experimental value (42 days) with CONRAD

\subsection{Experimental correlations and assimilation of several MERCI-1 experimental values}

In order to improve the data assimilation process, several experimental data could be used at the same time, using correlated fission yield data, for this study, given the positive results obtained in subsection 4.3. In this paragraph, measurements at different cooling times coming from the MERCI-1 experiment are taken into account simultaneously. In order to select which experimental data to use, the relative contribution of the fission products of interest are shown in Fig. 3. as a function of the cooling time.

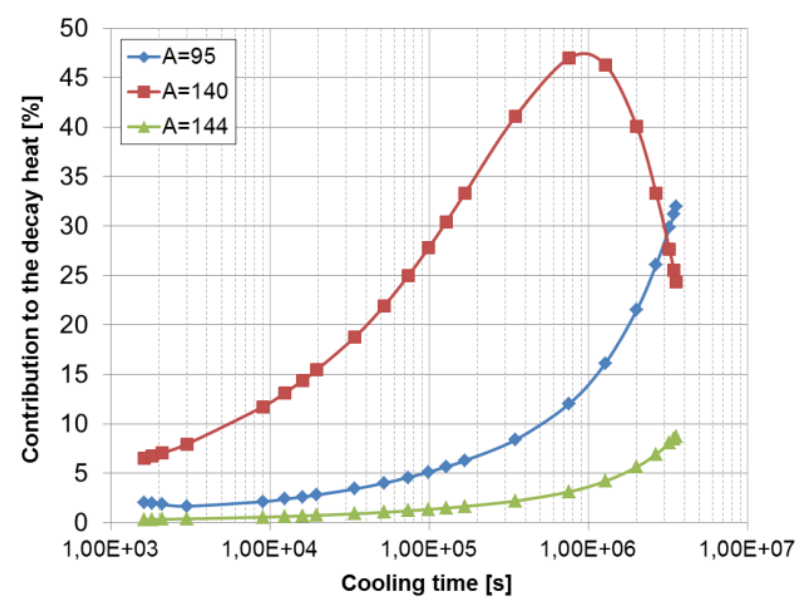

Fig. 3. Contributions of the main contributing masses to the decay heat [\%] over the cooling time [s] for the MERCI-1 experiment 
Judging from Fig. 3, it seems judicious to select cooling times rather close to the right side of the plot, i.e. cooling times above $10^{5} \mathrm{~s}$ for example, since the contributions of isotopes of masses 140 and 95 (a little less for mass 144) vary quite much on this range. Nine experimental values are then considered, corresponding to cooling times between 2 $\left(1.7 .10^{5} \mathrm{~s}\right)$ and 42 days (whose associated $(\mathrm{C} / \mathrm{E}-1)$ discrepancies are shown on Fig. 4).

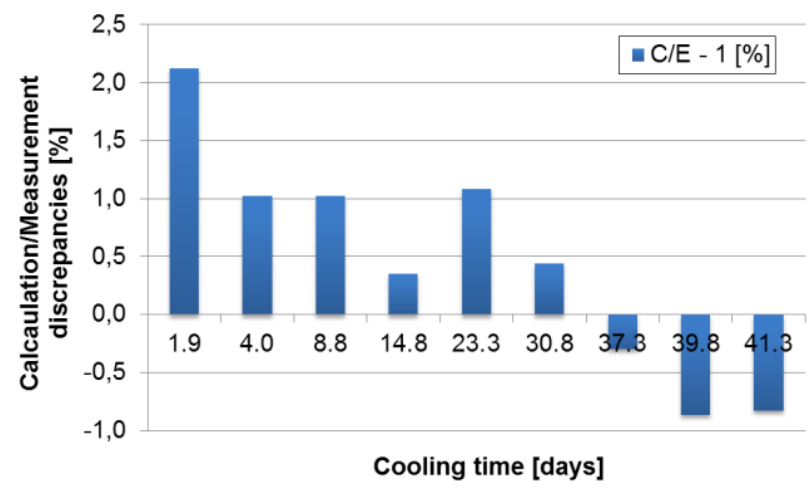

Fig. 4. MERCI-1 calculation/experiment discrepancies (C/E -1) for the nine cooling times considered

The simultaneous use of several experimental values coming from the same experiment raises indeed issues of experimental correlations to consider for the assimilation. Experimental correlations for each couple of experimental values $(a, b)$ were determined by calculating the covariance between each couple of measured decay heat values $\left(\mathrm{DH}_{\mathrm{a}}, \mathrm{DH}_{\mathrm{b}}\right)$ (at different cooling times) using equation (1) (cf. subsection 3.1). The resulting covariance is the following (3):

$$
\operatorname{cov}\left(\mathrm{DH}_{\mathrm{a}}, \mathrm{DH}_{\mathrm{b}}\right)=\alpha * \operatorname{cov}\left(\mathrm{Q}_{\mathrm{m}_{\mathrm{a}}}, \mathrm{Q}_{\mathrm{m}_{\mathrm{b}}}\right)+\beta * \operatorname{cov}\left(\mathrm{T}_{\mathrm{a}}, \mathrm{T}_{\mathrm{b}}\right)+\gamma * \operatorname{cov}\left(\mathrm{C}_{\mathrm{p}_{\mathrm{a}}}, \mathrm{C}_{\mathrm{p}_{\mathrm{b}}}\right)
$$

$\operatorname{cov}\left(\mathrm{T}_{\mathrm{a}}, \mathrm{T}_{\mathrm{b}}\right)$ is the covariance between temperatures, $\operatorname{cov}\left(\mathrm{Q}_{\mathrm{m}_{\mathrm{a}}}, \mathrm{Q}_{\mathrm{m}_{\mathrm{b}}}\right)$ is the covariance between two water mass flows and $\operatorname{cov}\left(\mathrm{C}_{\mathrm{p}_{\mathrm{a}}}, \mathrm{C}_{\mathrm{p}_{\mathrm{b}}}\right)$ is the covariance between water heat capacities. The two first covariance terms could not be assessed, due to a lack of information thereon in the experimental process. The last covariance term is determined by performing a polynomial regression $\left(3^{\text {rd }}\right.$ order) of the heat capacity against the temperature. The different coefficients of each power of the temperature in this polynomial are determined with associated uncertainties and covariances. It is thus possible to determine $\operatorname{cov}\left(\mathrm{C}_{\mathrm{p}_{\mathrm{a}}}, \mathrm{C}_{\mathrm{p}_{\mathrm{b}}}\right) \cdot \gamma * \operatorname{cov}\left(\mathrm{C}_{\mathrm{p}_{\mathrm{a}}}, \mathrm{C}_{\mathrm{p}_{\mathrm{b}}}\right)$ is numerically negligible in front of the two other terms because of the values of $\alpha, \beta$ and $\gamma$, involving $\mathrm{C}_{\mathrm{p}}$, $\mathrm{T}$ and $\mathrm{Q}_{\mathrm{m}}$ values.

The assimilation results with the use of the nine experimental values abovementioned are presented on Fig. 5. Different tests were performed with different experimental correlation values to try and measure the sensitivity of the assimilation results due to the experimental correlations considered between each couple of decay heat values $(0.1,0.5,0.8$ and 0.9 , cf. Fig.5). The trends obtained with CONRAD for each independent fission yield are consistent at 1 std with what is planned for JEFF-3.3, regardless of the experimental correlations considered. The sensitivity of the assimilation results due to the experimental correlations considered is lower than the sensitivity due to the inclusion of the correlations between iFY. However, depending on the iFY studied, the trends obtained with the simultaneous use of the nine experimental values get closer to JEFF-3.3 with lower experimental correlations for ${ }^{144} \mathrm{Ba},{ }^{140} \mathrm{Xe},{ }^{95} \mathrm{Sr}$ and ${ }^{95} \mathrm{Y}$, but with higher correlations for ${ }^{144} \mathrm{La}$ and ${ }^{140} \mathrm{Cs}$, hence the importance of ensuring the experimental correlations to consider, 
to be able to propose real feedbacks on nuclear data when using more than a single experiment.

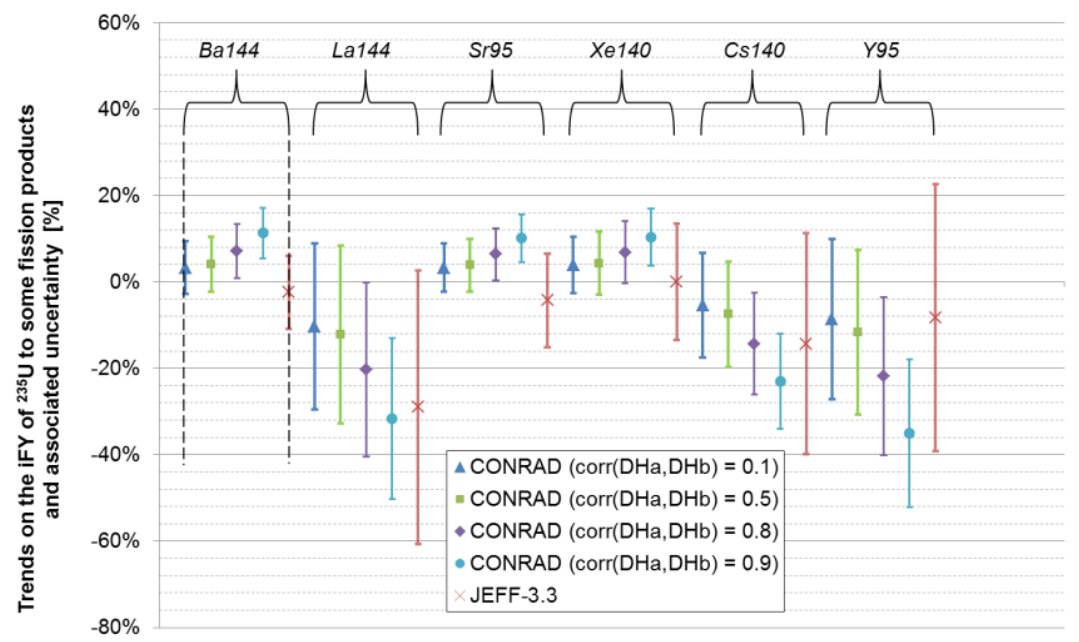

Fig. 5. Assimilation of nine correlated MERCI-1 experimental values (with different correlations)

\section{Conclusions}

The assimilation studies presented in this paper show that assimilating several correlated experimental values (instead of a single one) and adding covariance information on independent fission yield data of ${ }^{235} \mathrm{U}$ result in trends (on nuclear data of interest for the decay heat computation) in better accordance with JEFF-3.3. Even if it was not possible yet to determine the actual experimental correlations between the different MERCI-1 experimental values at different cooling times, it was possible to carry out a sensitivity study of the assimilation results obtained for several experimental correlation test values. It can be noticed that the results are in better accordance with JEFF-3.3 regardless of the experimental correlations considered between each couple of decay heat measurements. Other studies over the simultaneous assimilation of different integral experimental data and/or fission burst experiments could be performed, to verify the consistency between the different feedbacks thus obtained on nuclear data of interest for the decay heat computation.

\section{References}

1. J.C. Jaboulay, S. Bourganel, Nuclear Technology, 177, 73-82 (2012)

2. N. Terranova, Covariance evaluation for nuclear data of interest to the reactivity loss estimation of the Jules Horowitz Material Testing Reactor (PhD thesis, 2016)

3. Ch. Blandin et al., International Group Operating Research Reactors IGORR12 (2009)

4. C. De Saint Jean et al., Int. Conf. On Nuclear Data for Science and Technology (2007)

5. L. San Felice et al., Nuclear Technology, 184, 217-232 (2013)

6. A. Santamarina et al., JEFF Report 22 NEA No. 6807 OECD (2009)

7. OECD, JEFF-3.3, https://www.oecd-nea.org/dbdata/jeff/jeff33/

8. P. Archier et al., Proc. Int. Conf. PHYSOR (2014) 